\title{
Structural Basis of Light Harvesting by Carotenoids: Peridinin-Chlorophyll-Protein from Amphidinium carterae
}

\author{
Eckhard Hofmann, Pamela M. Wrench, Frank P. Sharples, \\ Roger G. Hiller, Wolfram Welte, ${ }^{*}$ Kay Diederichs
}

Peridinin-chlorophyll-protein, a water-soluble light-harvesting complex that has a bluegreen absorbing carotenoid as its main pigment, is present in most photosynthetic dinoflagellates. Its high-resolution (2.0 angstrom) $x$-ray structure reveals a noncrystallographic trimer in which each polypeptide contains an unusual jellyroll fold of the $\alpha$-helical amino- and carboxyl-terminal domains. These domains constitute a scaffold with pseudo-twofold symmetry surrounding a hydrophobic cavity filled by two lipid, eight peridinin, and two chlorophyll a molecules. The structural basis for efficient excitonic energy transfer from peridinin to chlorophyll is found in the clustering of peridinins around the chlorophylls at van der Waals distances.

Light-harvesting complexes (LHCs) increase the overall efficiency of photosynthesis by passing absorbed light to reaction centers, where conversion to chemical energy occurs. Photosynthetic dinoflagellates, which make up much of the sea plankton and are the cause of red tides, use about equal amounts of two classes of pigments, carotenoids (1) and chlorophyll, for the efficient harvesting of light. Most dinoflagellates have peridinin (Fig. 1) as their predominant carotenoid, enabling them to capture solar energy in the blue-green range (470 to $550 \mathrm{~nm}$ ), which is inaccessible by chlorophyll alone. In addition to a membrane-bound LHC (2), which structurally and functionally resembles that of higher plants (3), dinoflagellates have developed a soluble antenna with a high carotenoid:chlorophyll ratio, peridinin-chlorophyll-protein (PCP), that has no sequence similarity with other known proteins (4). Variants of PCP with an apoprotein of around $16 \mathrm{kD}$ exist as homodimers (5), whereas forms with an apoprotein of around $32 \mathrm{kD}$ probably arose by duplication and fusion of an ancient PCP gene. In PCP from Amphidinium carterae, the $\mathrm{NH}_{2}$ - and $\mathrm{COOH}$-terminal domains (6) of the cDNAderived sequence $(30.2 \mathrm{kD})$ share $56 \%$ of their residues, and each domain binds a cluster of one chlorophyll $a$ and four peridinin molecules. Within each cluster, the efficiency of singlet energy transfer from peridinin to chlorophyll is close to unity (7). Models of chromophore interaction within and among the clusters have previously been based on spectroscopic investigations $(7,8)$. Structural

$\overline{\text { E. Hofmann, W. Welte, K. Diederichs, Fakultät für Biolo- }}$ gie, Universität Konstanz, Postfach 5560 (M656) , 78434 Konstanz, Germany.

P. M. Wrench, F. P. Sharples, R. G. Hiller, School of Biological Sciences, Macquarie University, New South Wales 2109, Australia.

*To whom correspondence should be addressed. E-mail: Wolfram.Welte@uni-konstanz.de phy. Diffraction data and crystallographic procedures are summarized in Table 1. Initial phases at $2.9 \AA$ resolution were obtained by the single isomorphous replacement method and improved by an iterative solvent-flattening and noncrystallographic symmetry-averaging procedure. The resulting electron density map enabled us to trace three crystallographically independent but nearly identical monomers (312 residues) of PCP and to insert the pigments. After extending the resolution to $2.0 \AA$ and refining further, lipids and water molecules were found in difference Fourier maps and were inserted into the model. The lipids are an integral part of the structure; their existence in PCP was unexpected and points to the complexity of the holoprotein assembly. On the basis of welldefined density and possible hydrogen bonds, the lipids were identified (Table 1) as digalactosyl diacyl glycerol (DGDG). We assume that DGDG binds tightly to the chlorophylls before complex formation; its removal during unfolding by acetone may cause the failure of renaturation experiments (7). As DGDG is mostly found in the inner thylakoid membrane (11), where it is the main lipid, our finding supports the disposition of PCP inside the thylakoid lumen, which was previously based only on an analysis of leader sequences (4).

In the crystal structure (Fig. 2), the $\mathrm{NH}_{2}$ - and $\mathrm{COOH}$-terminal halves of the polypeptide form almost identical domains related by a twofold pseudosymmetry axis [root

Table 1. Crystallographic data. Data collection: Native1 and derivative data (5 $\mathrm{mM} \mathrm{K}_{2} \mathrm{PtCl}_{4}, 1$-day soak) were collected on a rotating anode source (CuK $\alpha, 40 \mathrm{kV}, 100 \mathrm{~mA}$ ) with a STOE (Darmstadt, Germany) imaging plate detector. Native2 was collected at the BW7B wiggler beamline at DESY on a $30-\mathrm{cm}$ MARresearch (Hamburg, Germany) image plate. All data were processed with XDS (26). Phasing: Six heavy-atom binding sites were found using SHELXS (27) in Patterson search mode, and four additional sites were found by inspection of difference Fourier maps. Refinement of heavy-atom parameters was done with DAREFI (28). The phases could be improved further by inclusion of the anomalous signal and the use of solvent flattening and noncrystallographic symmetry averaging of the trimer in the asymmetric unit (29). Model building and refinement: The resulting $2.9 \AA$ electron density map was readily interpretable. An initial model of PCP was built with $O$ (23). The full sequence could be included, and 10 pigments were modeled. The initial model was refined with X-PLOR (24), including simulated annealing, positional refinement, and manual rebuilding against Native1 and later Native2. Strong noncrystallographic symmetry restraints were applied throughout the refinement. ARP $(30)$ was used to obtain unbiased atomic positions for well-connected density in the $2.0 \AA$ difference electron-density map. The arrangement of these atoms, together with possible hydrogen-bonding patterns, was consistent with an interpretation as DGDG molecules. After refinement, the DGDG head groups obey good stereochemistry and show no difference density. The current model consists of 6849 protein atoms, 1890 atoms of pigments and lipids, and 416 water atoms, and the $R$, $\left[=\Sigma_{1}\left|F_{0}-F_{\mathrm{c}}\right| / \Sigma_{h} F_{0}\right.$, where $F_{0}$ and $F_{\mathrm{c}}$ are the observed and calculated structure factor amplitudes; summation is over all reflections $h]$ in the resolution range 40 to $2 \AA$ is $17.9 \%$ for 93,720 reflections [free $R(24), 20.1 \%$ for 2325 reflections]. Stereochemical deviations from target values are $0.012 \AA$ for bond lengths and $1.3^{\circ}$ for bond angles. No residues are in disallowed regions of the Ramachandran plot.

\begin{tabular}{lccccc}
\hline Data & $\begin{array}{c}\text { Resolution }(\AA) \\
\text { (highest shell) }\end{array}$ & $\begin{array}{c}\text { Unique } \\
\text { reflections }\end{array}$ & $\begin{array}{c}\text { Completeness }(\%) \\
\text { (highest shell) }\end{array}$ & $\begin{array}{c}R_{\text {sym }}{ }^{*}(\%) \\
\text { (highest shell) }\end{array}$ & $\begin{array}{c}R_{\text {iso }}{ }^{\dagger} \\
\text { (\%) }\end{array}$ \\
\hline Native1 & $2.9(3.16-2.9)$ & 31362 & $93.6(82.4)$ & $8.3(26.5)$ & - \\
Native2 & $2.0(2.10-2.0)$ & 96045 & $92.5(92.6)$ & $5.4(19.0)$ & - \\
$\mathrm{K}_{2} \mathrm{PtCl}_{4}$ & $2.9(3.16-2.9)$ & 31384 & $93.6(58.6)$ & $11.9(41.1)$ & 33.4 \\
\hline
\end{tabular}

${ }^{*} R_{\text {sym }}=\Sigma_{h} \Sigma_{i}\left|I_{h, j}-\left\langle I_{h}\right\rangle\right| / \Sigma_{h} \Sigma_{i} I_{h, l}$, where $\left\langle I_{h}\right\rangle$ is the mean intensity of all observations of the unique reflection $h^{\text {sym }}+R_{\text {iso }}=\Sigma_{h}\left|I_{\mathrm{P}}-I_{\mathrm{PH}}\right| / \Sigma_{h i}\left(I_{\mathrm{P}}+I_{\mathrm{PH}}\right) / 2$, where $I_{\mathrm{P}}$ and $I_{\mathrm{PH}}$ are the intensities of the native and derivative data sets, respectively; summation is over all reflections $h$ present in both. 
mean square deviation of $0.9 \AA$ (12)]. Each domain consists of eight helices (N1 through $\mathrm{N} 8$ and $\mathrm{C} 1$ through $\mathrm{C} 8$ ) and their intervening loops, with a topology resembling a wide open, right-handed "jellyroll" (13); a short $\beta$-hairpin loop forms the tip of the jellyroll. The juxtaposition of these domains generates a central, hydrophobic space of about $23 \AA$ by $23 \AA$ by $53 \AA$ filled with the pigments and lipids (holoprotein molecular weight, $38.1 \mathrm{kD})$. This jellyroll fold has not previously been reported (14) for an $\alpha$-helical protein. The fold appears to be advantageous, as it economizes on the number, size, and angular range of movements during folding of the protein around the chromophores after translocation through the chloroplast membranes. The shape of the protein, with its triangular cross section, can best be described by analogy with a ship, whose bow, sides, stern, and deck are formed by helices. This analogy can be extended to describe the long (40 $\AA$ ) connection (12) between the $\mathrm{NH}_{2}$ - and $\mathrm{COOH}$-terminal domains as the keel of the ship and the chromophores in the interior of the ship as the cargo. Two large openings are found near the bow and the stern, between helices N8 and N6 and between C8 and C6, and two smaller ones are found in the deck, between helices $\mathrm{N} 2$ and $\mathrm{N} 7$ and between $\mathrm{C} 2$ and $\mathrm{C} 7$. These openings are filled by the hydrophilic epoxycyclohexane rings of the peridinins $\left(\mathrm{NH}_{2}\right.$ - and $\mathrm{COOH}$-terminal Per1 to Per4) and the head groups of the lipids.

Monomers of PCP form a noncrystallographic trimer with a diameter of about $100 \AA$ and a thickness of $40 \AA$; their local twofold axes are tilted by $20^{\circ}$ with respect to the trimer plane. Trimerization occurs through mainly hydrophobic interactions of the deck and stern helices and is assisted by hydrogen bonds of the $\mathrm{COOH}$-terminal Per2 epoxycyclohexane ring, which interacts with its symmetry mates around the threefold axis through an intervening water molecule. Trimerization of PCP is consistent with dynamic light-scattering properties of concentrated PCP solutions. Furthermore, we solved by the molecular replacement method two

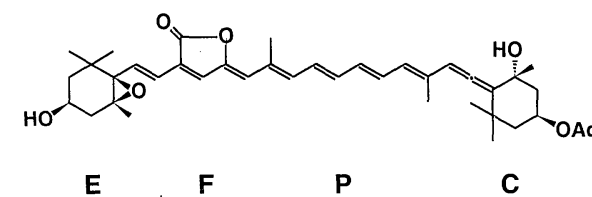

Fig. 1. Structure of peridinin, an asymmetric carotenoid composed of an epoxycyclohexane ring $(E)$ and a polyene chain $(P)$ with a furanic ring $(F)$ and a cyclohexane ring (C). Distortions of the alltrans configuration, as well as contacts of $\mathrm{P}$ and $\mathrm{F}$ with polar atoms of the protein environment, modify the spectroscopic properties [peridinin in ethanol, absorption maximum $\lambda_{\max }=470 \mathrm{~nm}$ (7); peridinin in peridinin-chlorophyll-protein (PCP), $\lambda_{\max }$ $=478 \mathrm{~nm}$. nonisomorphous structures of PCP crystals grown under significantly different conditions and observed the same mode of trimerization in these different environments (15). Trimer formation, which is also found in the other two structurally known watersoluble LHCs, bacteriochlorophyll a protein (16) and phycobiliprotein (17), as well as in LHC-II from higher plants (3), has been interpreted as advantageous for orienting the pigments and so optimizes the absorption cross section (16). It is striking that evolution has used two different structural classes, all- $\alpha$ and all- $\beta$, to build the water-soluble PCP and bacteriochlorophyll a protein, which have in common a hydrophobic space filled by a cluster of pigments.

In photosynthesis, energy is transferred (18) among similar or identical chromophores by two mechanisms. Long-range (up to $100 \AA$ ) Förster dipole-dipole inter- action depends strongly on the mutual orientation of the dipoles: it is most efficient if dipoles are parallel and end-to-end but is still favorable if they are parallel and sideby-side. Coupling of delocalized excitons occurs at distances of less than $20 \AA$. For a high efficiency of excitonic energy transfer between carotenoids and chlorophyll, much shorter distances are required, because of the short half-life of the carotenoid excited state (19). Circular dichroism studies of PCP (7) suggested two chlorophyll-peridinin clusters in which each chlorophyll is arranged between two pairs of mutually orthogonal peridinins. The crystal structure confirms the existence of a $\mathrm{NH}_{2}$-terminal (Fig. 3) and a $\mathrm{COOH}$-terminal pigment cluster, related by the same local twofold symmetry as is the apoprotein. Each cluster has two pairs of peridinins (Per1-Per2 and Per3-Per4) surrounding a chlorophyll; with-
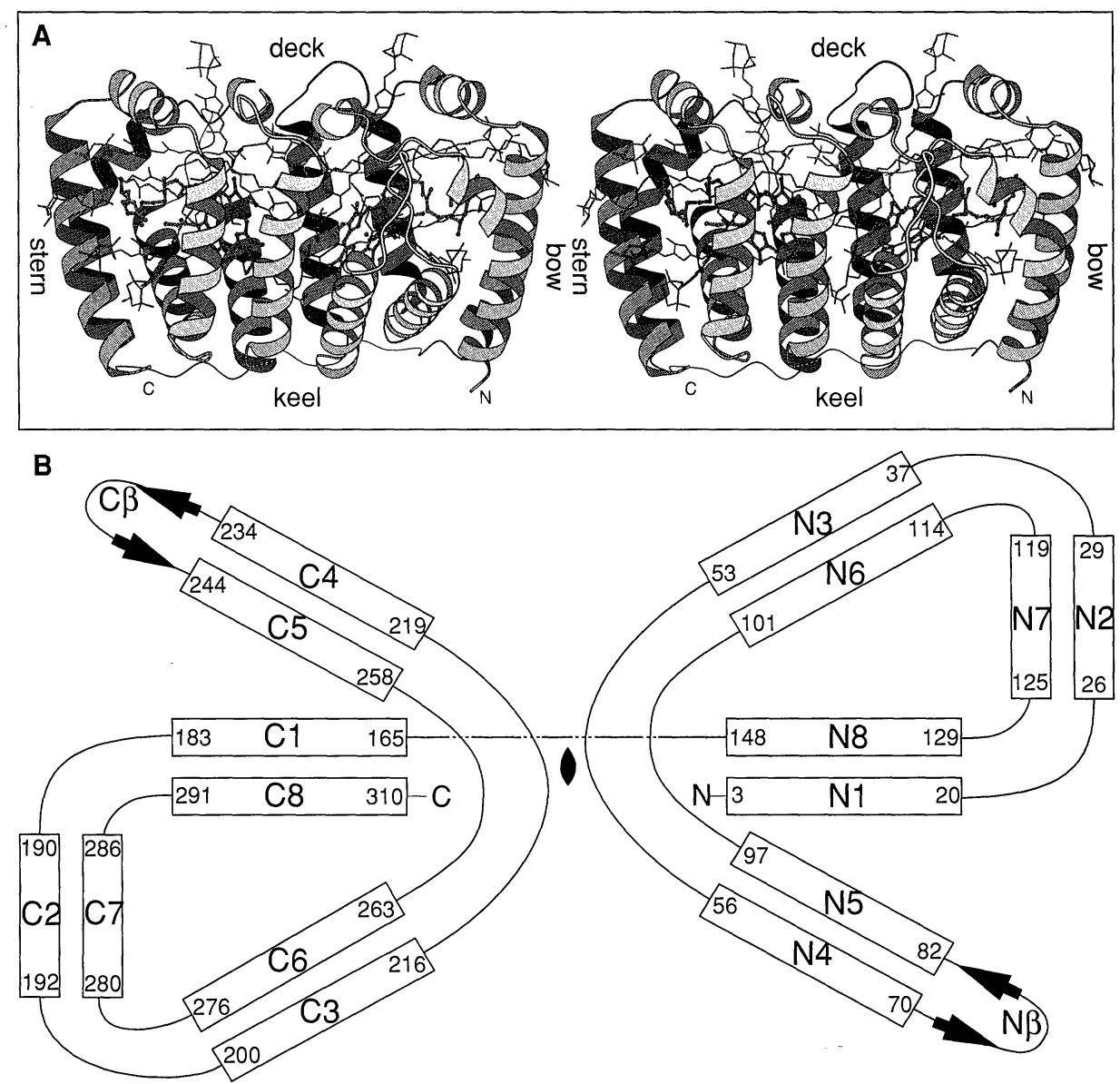

Fig. 2. Structure of PCP. (A) Stereo ribbon diagram of a monomer of the PCP complex. The $\mathrm{NH}_{2}$ terminus is positioned at the lower right of the diagram, the $\mathrm{COOH}$ terminus at the lower left. Helices are gray, chlorophyll molecules are green, peridinins are in shades of red (Per1, lightest, to Per4, darkest), and lipids are in blue. The helices form a scaffold resembling a ship, whose parts are labeled. The view is approximately along the trimer axis, and the local twofold pseudosymmetry axis, which relates the $\mathrm{NH}_{2}$ - and $\mathrm{COOH}$-terminal domains, is vertical. [Produced by MOLSCRIPT (25).] (B) Topology diagram of a PCP monomer. The jellyroll formed by the helices of each domain is depicted in a nonstandard (13) way, with $\mathrm{NH}_{2}$ and $\mathrm{COOH}$ termini in the center, thereby conserving the spatial relations between helices. The "ship" consists of the bow (N1 and N8), sides (N3 through N6 and C3 through C6), stern (C1 and $\mathrm{C} 8$ ), and deck (N2, N7, C2, and C7). Helices parallel in the diagram are almost parallel in the structure, whereas all other pairs of adjacent helices cross at angles of around $30^{\circ}$ to $50^{\circ}$. 
in each pair, closest distances of the polyene chains are less than $4 \AA$. All peridinins are in an extended all-trans conformation and show small distortions from their average geometry (20), which mainly result from interaction with the protein environment. In contrast to the predictions (7), the crossing angle within each pair is about $56^{\circ}$ $\left( \pm 6^{\circ}\right)$, the furanic rings of the peridinins are close to each other within each pair, and the distance between the centers of mass, which was estimated at $12 \AA(7)$, is $5.5 \AA$ (Per1-Per2) and $8.4 \AA$ (Per3-Per4), respectively. Per1 and Per 3 are parallel, to within $5^{\circ}$, and flank the tetrapyrrole system on opposite sides, extending a plane that is tilted by $30^{\circ}$ from that of chlorophyll (Fig. $3)$. The other members of each pair, Per2 and Per4, are stacked on opposite faces of the chlorophyll macrocycle and are within $25^{\circ}$ of the $Q_{x}$ and $Q_{y}$ transition moments (21) of chlorophyll, respectively. The conjugated regions of all peridinins are in van der Waals contact ( 3.3 to $3.8 \AA$ ) with the tetrapyrrole ring of chlorophyll, allowing efficient excitonic energy transfer from each peridinin to chlorophyll.

The chlorophylls are completely buried in a hydrophobic environment: half of their surface area is covered by the peridinins, and the remainder is covered by the protein (one-third) and the fatty acid chains (onesixth) of the lipids. The closest proteinchlorophyll contacts occur through a stacking at van der Waals distance of the imidazole rings of two conserved His residues, $\mathrm{His}^{66}$ and $\mathrm{His}^{229}$, onto the tetrapyrrole C ring of the $\mathrm{NH}_{2-}$ and $\mathrm{COOH}$-terminal chlorophylls, respectively. A water molecule, which is on one side hydrogen bonded to these residues, provides the fifth coordination site (distance, $2.0 \AA$ ) of the central Mg atoms (Fig. 4). In both clusters, chlorophyll intercalates into the pocket between the two pairs of peridinins, with its phytyl chain projecting into the space between Per1 and Per3. Unlike the situation in LH2 (9), where excitonic energy transfer between adjacent chlorophylls takes place, the geometry in PCP allows only Förster energy transfer between chlorophylls of each monomer (distance, $17.4 \AA$ ). Distanc-

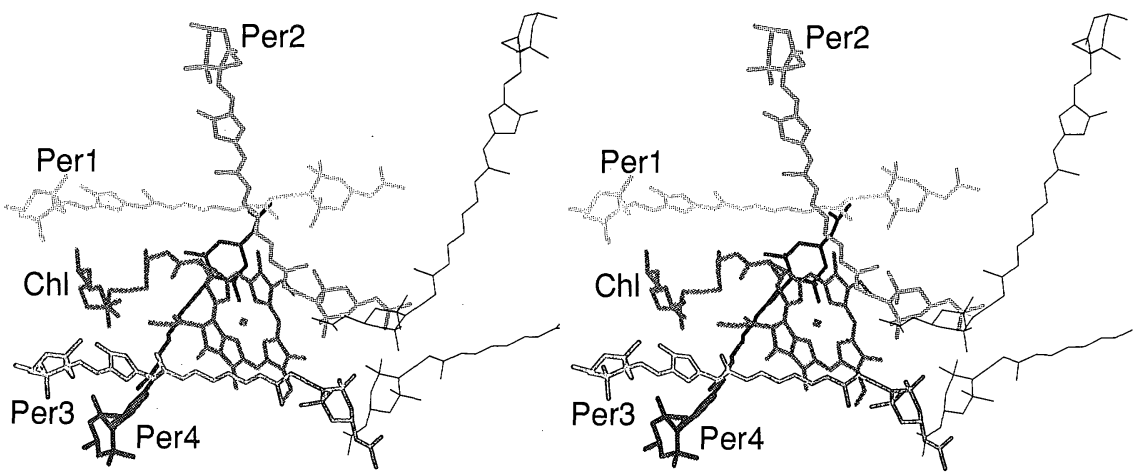

Fig. 3. Stereo view of the arrangement of peridinins and chlorophyll in the $\mathrm{NH}_{2}$-terminal cluster (heavy lines). Peridinins are labeled Per1 to Per4. Two peridinins (Per2 and Per3) of the $\mathrm{COOH}$-terminal pigment cluster are shown in black (thin lines). The view is the same as in Fig. 2A.

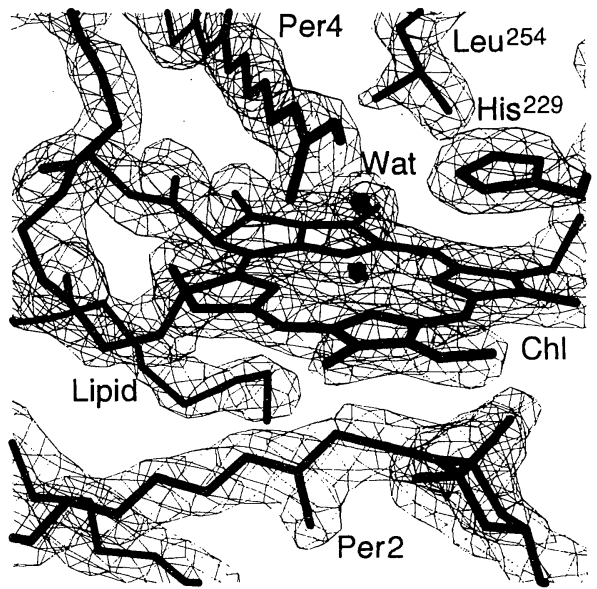

Fig. 4. Electron density map (coefficients $2 F_{0}-F_{\text {, }}$ contoured at $1.5 \sigma$ ) showing a stereo view of the environment of the $\mathrm{COOH}$-terminal chlorophyll (Chl), including Leu ${ }^{254}$, His ${ }^{229}$, and parts of Per2, Per4, and one lipid molecule. A water molecule (Wat), which provides the fifth coordination site of the $\mathrm{Mg}$, is also shown. The view is parallel to the trimer axis. es between chlorophylls of different subunits within a trimer are in the 40 to $55 \AA$ range. The $\mathrm{COOH}$-terminal chlorophylls appear to be oriented most suitably for Förster exchange of excitation energy between the subunits of a trimer (Fig. 5), as their $Q_{y}$ transition moments are approximately parallel to the trimer axis (inclination, $25^{\circ}$ ), and their distance $(44 \AA)$ is well within the range of dipole-dipole interactions.

The polypeptide chain of PCP serves three main purposes: (i) to provide a hydrophobic environment for the pigments, which would otherwise be insoluble in the aqueous compartment of the chloroplast, (ii) to tune the absorption properties of the pigments, and (iii) to arrange the pigments such that there is efficient energy transfer among them and toward the other components of the photosynthetic apparatus. PCP shares the latter two requirements with the membrane-bound LHC. The structure of LHC-II from higher plants has been elucidated by electron microscopy (3) and can serve as a structural model for other LHCs, including that of dinoflagellates, with which it shares about $30 \%$ sequence homology (2). Interestingly, we note structural similarities that parallel the functional similarities between these two proteins: Both are elongated monomers that associate to form flat trimers, and the trimer interface is in both cases provided primarily by one of the two monomer halves that is related to the other half by a local twofold axis. In a model based on fluorescence rise times, it is

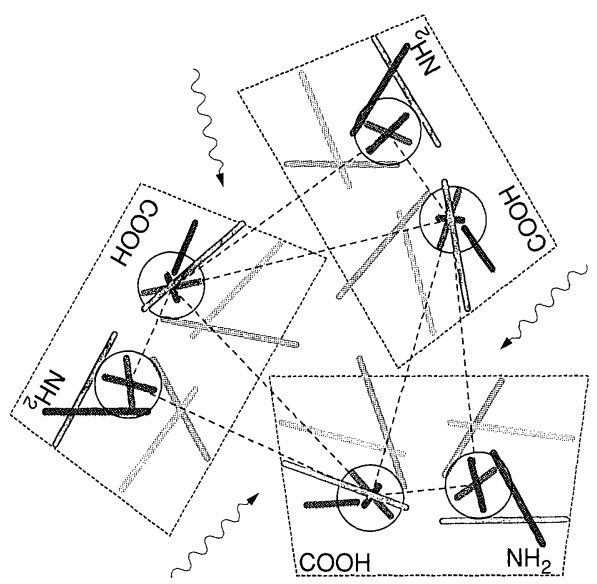

Fig. 5. Model of energy flow to, from, and within the PCP trimer (top view). Light absorbed by the polyene chains of peridinin (red;"color-coded as in Fig. 2A) is transferred by an excitonic mechanism to chlorophyll :(circled). Crosses represent the transition moments $\left(Q_{x}\right.$, light green; $Q_{y}$, dark green). Energy can be equilibrated (dashed lines) among the chlorophylls of the trimer or passed to membrane-bound chlorophylls (below or above the plane of the trimer) by a dipole-dipole mechanism. Individual monomers are delineated by dotted lines, and the $\mathrm{NH}_{2}$ and $\mathrm{COOH}$ termini are indicated. 
proposed (22) that PCP passes energy from its chlorophylls to those of the membranebound LHC. Although the data (22) do not exclude direct energy transfer to the core of photosystem 2, the similar appearance of the PCP trimer and that of the intrinsic chlorophyll-carotenoid protein suggests that PCP and LHC could coexist in a stacked configuration. With this proposed geometry, highly efficient Förster energy transfer from PCP to LHC can be expected, because the tetrapyrrole rings of their chlorophylls would be approximately coplanar.

\section{REFERENCES AND NOTES}

1. For recent reviews about the photoprotective and light-harvesting functions of carotenoids, see $\mathrm{H}$. A. Frank and R. J. Cogdell, in Carotenoids in Photosynthesis, A. Young and G. Britton, Eds. (Chapman \& Hall, London, 1993); Y. Koyama, M. Kuki, P. O. Andersson, T. Gillbro, Photochem. Photobiol. 63, $243(1996)$

2. R. G. Hiller, P. M. Wrench, A. A. Gooley, G. Shoebridge, J. Breton, Photochem. Photobiol. 57, 125 (1993); R. Iglesias-Prieto, N. S. Govind, R. K. Trench, Philos. Trans. R. Soc. London Ser. B 403, 381 (1993); R. G. Hiller, P. M. Wrench, F. P. Sharples, FEBS Lett. 363, 175 (1995)

3. W. Kühlbrandt and D. N. Wang, Nature 350,130 (1991); and Y. Fujioshi, ibid. 367, 614 (1994).

4. B. J. Norris and D. J. Miller, Plant Mol. Biol. 24, 673 (1994).

5. F. T. Haxo, J. H. Kycia, G. F. Somers, A. Bennett, H. W. Siegelman, Plant Physiol. 57, 297 (1976); B. B. Prezelin, in The Biology of Dinoflagellates, F. J. R. Taylor, Ed. (Blackwell Scientific, Oxford, 1987), p. $174 ;$ R. Iglesias-Prieto, N. S. Govind, R. K. Trench, Proc. R. Soc. London Ser. B 246, 275 (1991); E. L. Triplett et al., Mol. Mar. Biol. Biotechnol. 2, 246 (1993).

6. R. G. Hiller, P. M. Wrench, F. P. Sharples, in Photosynthesis: From Light to Biosphere, P. Mathis, Ed. (Kluwer, Dordrecht, Netherlands, 1995), vol. 1, p. 24.

7. P. S. Song, P. Koka, B. B. Prezelin, F. T. Haxo, Biochemistry 15, 4422 (1976); P. Koka and P. S. Song, Biochim. Biophys. Acta 495, 220 (1977).

8. D. Carbonera, G. Giacometti, G. Agostini, Spectrochim. Acta A 51, 115 (1995).

9. G. McDermott et al., Nature 374, 517 (1995).

10. Purification and crystallization: A. carterae was cultivated as reported previously (2), and PCP was purified from a water-soluble algal extract by size-exclusion chromatography and chromatofocusing. Crystals grew in the monoclinic space group C2 with cell dimensions of $a=198.4 \AA, b=116.3 \AA, c=67.0 \AA$ and $\beta=94.9^{\circ}$. $\mathrm{PCP}$ is present as a trimer with an overall weight-average molecular weight of $114 \mathrm{kD}$ in the asymmetric unit. The absorption spectrum is unchanged by the crystallization process. PCP crystals were grown at $17^{\circ} \mathrm{C}$ in hanging drops containing $5 \mathrm{mg}$ of protein per milliliter, 4 to $6 \%$ PEG8000 (PEG, polyethylene glycol) in the crystallization buffer $\left[100 \mathrm{mM} \mathrm{MgCl}_{2}, 50 \mathrm{mM} \mathrm{KCl}, 24 \mathrm{mM}\right.$ triethylammoniumphosphate buffer, and $50 \mathrm{mM}$ tris$\mathrm{HCl}(\mathrm{pH} 5.8)$ or $100 \mathrm{mM} \mathrm{MgCl}_{2}, 50 \mathrm{mM} \mathrm{KCl}$, and $25 \mathrm{mM}$ MES-KOH $(\mathrm{pH} 5.8)]$ with a reservoir of 8 to $12 \%$ PEG8000. For heavy-atom screening, crystals were transferred to droplets of equivalent PEG concentration in MES crystallization buffer containing the heavy-atom compound.

11. A. Rawlyer, M. Meylan-Bettex, P. A. Siegenthaler, Biochim. Biophys. Acta 1233, 122 (1995).

12. Using a distance cutoff of $3.8 \AA$ in the program 0 (23), we aligned $149 \mathrm{C}$ atom pairs. Residues 151 through 163 are in extended conformation and connect the $\mathrm{NH}_{2}$ - and $\mathrm{COOH}$-terminal halves; consequently, they do not obey twofold local symmetry.

13. J. Richardson, Adv. Protein Chem. 34, 167 (1981).

14. Structural comparisons against databases of unique structures were performed with two different programs: DALI [L. Holm and C. Sander, J. Mol. Biol.
233, 123 (1993)] and SUPERIMPOSE [K. Diederichs, Proteins Struct. Funct. Genet. 23, 187 (1995)].

15. These two other crystal forms of PCP were obtained by a different purification scheme involving ammonium sulfate precipitation [K. Steck, T. Wacker, W. Welte, F. P. Sharples, R. G. Hiller, FEBS Lett. 268, 48 (1990)]. Data for space group P1 were collected to $2.7 \AA$ resolution from one crystal on a RAXIS IIc image plate detector at Molecular Structure Corporation (Houston, TX). Data for space group C2 with cell axes different from (10) were measured from one crystal on a FAST area detector at CNRS (Grenoble, France) to a maximum resolution of $3.2 \AA$. The crystal structures were solved by the molecular replacement procedures as implemented in the program X-PLOR (24). In both cases, we used a trimer of PCP as the search model and obtained unambiguous solutions of the rotation and translation functions. After rigid body refinement, the $R$ factor was less than $30 \%$ for both crystal forms. The correctness of the molecular replacement solutions was confirmed by omit maps showing the chlorophyll molecules, which had been left out of the model used for structure factor calculation.

16. B. W. Matthews, R. E. Fenna, M. C. Bolognesi, M. F. Schmid, J. M. Olson, J. Mol. Biol. 131, 259 (1979).

17. T. Schirmer, W. Bode, R. Huber, W. Sidler, H. J Zuber, ibid. 184, 257 (1985).

18. For a review, see T. Förster, in Modern Quantum Chemistry, Istanbul Lectures, Part III: Action of Light and Organic Crystals, O. Sinanoglu, Ed. (Academic Press, New York, 1965), pp. 93.

19. Estimates of distances permitting efficient energy transfer from peridinins to chlorophyll were given as at most 5.8 to $8.6 \AA(7)$, approximately $5.0 \AA(22)$ and $4.5 \AA$ [T. Gillbro et al., Photochem. Photobiol. 57, 44 (1993)].

20. Pairwise comparisons give root-mean-squares deviations between 0.6 and $1.3 \AA$
21. The direction of the $Q_{y}$ transition moment was taken as the vector between the $\mathrm{C} 1 \mathrm{~B}$ and $\mathrm{C} 2 \mathrm{D}$ atoms of the porphyrin ring [nomenclature as in D. E. Tronrud, M. F. Schmid, B. W. Matthews, J. Mol. Biol. 188, 443 (1986)].

22. M. Mimuro, N. Tamai, T. Ishimaru, I. Yamazaki, Biochim. Biophys. Acta 1016, 280 (1990)

23. T. A. Jones, J. Y. Zou, S. W. Cowan, M. Kjeldgaard, Acta Crystallogr. A 47, 110 (1991).

24. A. T. Brünger, X-PLOR Version 3.1 (Yale Univ. Press, New Haven, CT, 1987); Nature 355, 472 (1992).

25. P. Kraulis, J. Appl. Crystallogr. 24, 946 (1991).

26. W. Kabsch, ibid. 21, 916 (1988).

27. G. M. Sheldrick, Acta Crystallogr. A 46, 467 (1990).

28. R. E. Dickerson, J. E. Weinzierl, R. A. Palmer, Acta Crystallogr. B 24, 997 (1968); K. Diederichs, Jt CCP4 ESF-EACBM Newsl. Protein Crystallogr. 31 23 (1994).

29. W. Furey and S. Swaminathan, in Methods Enzymol. in press

30. V. S. Lamzin and K. S. Wilson, Acta Crystallogr. D 49, 127 (1993).

31. We thank the staff of the European Molecular Biology Laboratory at the Deutsches Elektronen-Synchrotron (DESY) (Hamburg, Germany) for help during synchrotron data collection and the staff of Molecular Structure Corporation (Houston, TX) for the opportunity to collect $\mathrm{x}$-ray data of a $P 1$ crystal during a demonstration. We also thank K. Steck and P. Timmins for help in the early stages of the project, P. A Karplus for comments on the manuscript, and $W$. Kreutz for support. This work was supported by grants from the Deutsche Forschungsgemeinschaft and the Australian Research Council. The atomic coordinates have been submitted to the Brookhaven protein database (ID code 1PPR).

11 March 1996; accepted 23 April 1996 